\title{
Diagnostic benefits of the combined use of liquid-based cytology, cell block, and carcinoembryonic antigen immunocytochemistry in malignant pleural effusion
}

\author{
Chang Gok Woo ${ }^{1 \#}$, Seung-Myoung Son ${ }^{1 \#}$, Hye-Suk Han ${ }^{2,3}$, Ki Hyeong Lee ${ }^{2,3}$, Kang-Hyeon Choe ${ }^{2,3}$, \\ Jin Young $\mathrm{An}^{2,3}$, Ki Man Lee, ${ }^{2,3}$ Young Hyun Lim ${ }^{3}$, Ho-Chang Lee ${ }^{1,3}$, Ok-Jun Lee ${ }^{1,3}$ \\ ${ }^{1}$ Department of Pathology and ${ }^{2}$ Department of Internal Medicine, Chungbuk National University Hospital, ${ }^{3}$ Chungbuk National University College \\ of Medicine, Cheongju, Korea \\ Contributions: (I) Conception and design: CG Woo, SM Son, OJ Lee; (II) Administrative support: OJ Lee; (III) Provision of study materials \\ or patients: HS Han, KH Lee, KH Choe, JY An, KM Lee; (IV) Collection and assembly of data: CG Woo, YH Lim; (V) Data analysis and \\ interpretation: CG Woo, SM Son, HC Lee, OJ Lee; (VI) Manuscript writing: All authors; (VII) Final approval of manuscript: All authors. \\ \#These authors contributed equally to this work. \\ Correspondence to: Ok-Jun Lee, MD, PhD. Department of Pathology, Chungbuk National University Hospital, Chungbuk National University \\ College of Medicine, Chungdae-ro 1, Seowon-gu, Cheongju 28644, Korea. Email: ojlee@chungbuk.ac.kr.
}

\begin{abstract}
Background: Malignant pleural effusion (MPE) is a common complication of cancer cell metastasis to the pleura. Discrimination between MPE and benign pleural effusion is necessary to design treatment strategies. Cytology is important for the diagnosis of MPE. Carcinoembryonic antigen (CEA) is an epithelial biomarker with a strong staining pattern in adenocarcinomas. Here, the diagnostic performances of liquid-based cytology (LBC), cell block (CB) preparation, and CEA immunostaining for the detection of malignancy in effusion cytology were compared in a large case series.

Methods: In a single institution, 1,014 cytology samples from 862 patients were retrospectively collected and reviewed between January 2013 and November 2015. Ethanol-fixed, paraffin embedded CB of pleural effusions was analyzed by CEA immunostaining. Diagnostic values were compared among LBC, CB, CEA immunostaining, and the combination of two methods.

Results: The sensitivity and specificity of the CB preparation were $94.3 \%$ and $98.7 \%$, respectively, compared with $81.3 \%$ and $99.4 \%$ for LBC preparations, respectively. Combination of LBC and CB increased sensitivity by $98.3 \%$. Although the accuracy of CEA staining itself was moderate (sensitivity, $89.8 \%$ ), the combined use of CB and CEA tumor marker increased the detection rate of malignancy (sensitivity, 100\%; specificity, 100\%), compared with that of cytology (LBC or CB) alone.
\end{abstract}

Conclusions: The sensitivity and specificity for the diagnosis of MPE could be improved by integrating the $\mathrm{CB}$ and $\mathrm{CEA}$ staining into $\mathrm{LBC}$ in routine clinical practice to improve diagnostic accuracy.

Keywords: Cytology; cell block (CB); immunocytochemistry; carcinoembryonic antigen (CEA); Malignant pleural effusion (MPE)

Submitted May 15, 2018. Accepted for publication Jul 27, 2018.

doi: $10.21037 /$ jtd.2018.07.139

View this article at: http://dx.doi.org/10.21037/jtd.2018.07.139

\section{Introduction}

Malignant pleural effusion (MPE), a common complication of cancer cell metastasis to the pleura, is characterized by the proliferation of cancer cells in the pleura leading to abnormal fluid collection, classified as exudate according to Light's criteria $(1,2)$. Lung cancer is the most common primary tumor associated with MPE, followed by breast cancer $(3,4)$. Many patients with MPE have a history of 
primary cancer at different sites, but the pathological diagnosis is not always straightforward. In addition, pleural effusion may be the initial manifestation of cancer of unknown primary origin (5). The presence of malignant cells in the pleural effusion is associated with a poor prognosis (5). However, some cancer patients may show paramalignant pleural effusion, which refers to an effusion that does not result from neoplastic involvement of the pleura, despite being related to the primary tumor (6). For example, comorbidities such as post-obstructive pneumonia, obstruction of the thoracic duct, pulmonary embolism, or heart failure can produce an effusion, classified as transudate according to Light's criteria (2). Accordingly, the discrimination between MPE and benign pleural effusion is necessary to establish the etiology of the disease and design adequate treatment strategies.

Cytology plays a role in the diagnosis as well as in staging and in guiding the treatment of patients with MPE (7). The diagnostic rates of cytology in MPE range from $40 \%$ to $87 \%(4,7,8)$. Liquid-based cytology (LBC) is accepted as routine practice for the detection of cancer cells in patients with advanced cancer $(4,8-10)$. Although LBC is a highly specific diagnostic tool with up to $100 \%$ specificity, its sensitivity is limited (4). Distinguishing malignant from benign cells is a diagnostic challenge. For example, actively dividing mesothelial cells can overlap with carcinoma cells regarding morphologic features (11). These considerations have driven the introduction of ancillary tests to increase the diagnostic accuracy of cytology. The residual materials after LBC processing for cytological examination can be used in the preparation of cell blocks (CBs) (5). The CB technique is simple, non-invasive, and reproducible, and has a high yield for MPE. Moreover, the CB method is similar to hematoxylin and eosin (H\&E) staining of tissue specimens, and provides material for immunocytochemistry $(12,13)$. $\mathrm{CB}$ immunocytochemistry is accepted as an indispensable adjunct to cellular morphology in cytology $(14,15)$. It has the potential to improve the diagnostic accuracy of pleural effusion cytology in patients who are suspected of having cancer and to differentiate between reactive atypical cells and malignant cells.

Carcinoembryonic antigen-related cell adhesion molecule 5 (CEACAM5), also called CEA, may promote tumor development and the metastasis of cancer cells. CEA is a glycoprotein that is used as an epithelial biomarker with strong immunostaining in adenocarcinomas. Immunocytochemistry for CEA is $61-91 \%$ sensitive for the diagnosis of MPE in patients with metastatic adenocarcinoma. Although many studies have investigated tumor markers and compared cytology and CB technology for the diagnosis of MPE, most studies included a relatively small number of cases and focused on the accuracy of tumor markers $(12,13,16-22)$. To the best of our knowledge, no large-scale studies comparing $\mathrm{LBC}, \mathrm{CB}$, and the tumor marker CEA have been reported to date.

The purpose of the present study was to identify a method with optimal diagnostic accuracy in pleural effusions. Here, we compared the diagnostic performance of LBC, CB preparations, and CEA immunostaining for the detection of malignancy in effusion cytology in a large series of cases and discussed the diagnostic value of the addition of the tumor marker to cytological examination in clinical practice.

\section{Methods}

\section{Sample collection}

A total of 1,014 cytology samples from 862 patients who had undergone tapping of pleural effusion were retrospectively collected from the archives of the Department of Pathology at Chungbuk National University Hospital (Cheongju, Korea) between January 2013 and November 2015. Inadequate samples including scanty cellularity and obscuring blood were excluded from the study (Figure S1, CONSORT chart). Immunocytochemical staining for CEA using CB was performed in 131 cases $(12.5 \%)$ in which malignancy was suspected or the diagnosis was not clear. Clinicopathological data were obtained by reviewing medical records. The present study adhered to the guidelines established by the Declaration of Helsinki and was approved by the Institutional Review Board of Chungbuk National University Hospital (2014-02-009).

\section{Sample processing}

Pleural fluid samples were divided into equal parts for LBC and CB preparations. First, the samples were processed to produce LBC slides using CellprepPlus ${ }^{\circledR}$ (Biodyne, Seong-nam, Korea) following the manufacturer's protocol for sample preservation and slide preparation. Briefly, after fluid collection, samples were prepared by cytocentrifugation for $10 \mathrm{~min}$ at 2,000 rpm; the supernatant was decanted, the remaining pellet was treated with preservation liquid, and the preservation liquid was placed onto the CellprepPlus ${ }^{\circledR}$ device. Cells were filtered out with 
the CellprepPlus ${ }^{\circledR}$ and collected on the sides of the vessel using the blowing method. After smearing was completed, the slides were transferred automatically to fixing alcohol. CBs were generated by transferring an equal amount of fluid as that used for LBC to a suitable container and centrifugation for $10 \mathrm{~min}$ at $1,800 \mathrm{rpm}$. The supernatant was discarded, and the pellet was mixed with $95 \%$ ethanol solution and centrifuged again. The cell button obtained was cut to a suitable size, transferred to a cassette, and processed by three changes of alcohol, two changes of xylene, and two changes of paraffin to dehydrate-clearpenetrate the paraffin, yielding the CB. Sections were cut using a microtome (Leica Biosystems RM 2245, Nussloch, Germany) and stained with H\&E.

\section{Immunocytochemical staining for CEA}

Immunocytochemical staining of CEA proteins was performed in ethanol-fixed, paraffin embedded CB of pleural effusions. Immunocytochemistry was performed on $4 \mu \mathrm{m}$ CB paraffin sections using the Benchmark XT autostainer (Ventana Medical Systems, USA). Epitope retrieval was performed using the CC1 solution (Tris-EDTA buffer, pH 8.4; Ventana) for CEA. The slides were treated with $3 \%$ hydrogen peroxide for $4 \mathrm{~min}$ at $37.8{ }^{\circ} \mathrm{C}$ to block endogenous peroxidase activity and rinsed between steps with Ventana Tris-based reaction buffer. The slides were incubated with primary antibodies against CEA (12-140-10, 1:160; Novocastra) for 40 min followed by Ventana Universal HRP Multimer ( $8 \mathrm{~min}$ at $37.8^{\circ} \mathrm{C}$ ). Diaminobenzidine (DAB) was used as a chromogen, and hematoxylin was used as a nuclear stain. Immunoreactions were detected using the Ultraview Universal DAB detection kit (Ventana). The reactions were visualized with 3,3'-DAB, and the slides were counterstained with hematoxylin. Appropriate positive controls were used for each antibody.

\section{Cytological diagnosis}

LBC slides and $\mathrm{H} \& \mathrm{E}$-stained $\mathrm{CB}$ slides were retrospectively and blindly reviewed by two pathologists (OJ Lee and CG Woo), who were unaware of the clinical and pathological features. For each sample, the reviewers first examined the LBC slides, followed by the CB slides and immunocytochemical slides without consideration of the results obtained with the LBC slides. Immunostaining for CEA was scored as negative or positive. Cytoplasmic staining of more than $5 \%$ of atypical cells was considered positive, regardless of the intensity.

Cytological diagnoses were categorized into four groups as (I) negative (class I and II), (II) atypical (class III), (III) suspicious (class IV), and (IV) malignant (class V). The definition of "diagnosis confirmed malignant" was based on (I) malignant cells present in LBC and/or CB slides and a primary lesion pathologically and/or clinically confirmed as "malignant"; and (II) malignant cells confirmed by immunocytochemical staining. The definition of "diagnosis confirmed benign" was based on the absence of malignant cells observed by LBC and/or CB in samples from patients who had no clinical and/or radiological evidence of malignancy. Cases in which the primary cancer was histologically confirmed but effusion cytology identified no malignant cells were excluded.

\section{Comparison of diagnostic accuracy}

We checked the discrepancy among the methods including $\mathrm{LBC}, \mathrm{CB}$, the combination of $\mathrm{LBC}$ and $\mathrm{CB}$, CEA immunostaining, and the combined use of $\mathrm{CB}$ and CEA immunostaining. Based on the confirmed diagnosis, we matched the results of each method against the final diagnosis and investigated the sensitivity, specificity, positive predictive value, and negative predictive value of the each cytological method, except for cases classified as atypical.

\section{Results}

\section{Diagnostic distribution}

During 3 consecutive years, 862 patients underwent one or more thoracentesis at our institution for the diagnosis of PE (1,014 cases). Concurrent processing for LBC and CB was performed in the majority of cases (84.9\%), and CEA immunocytochemistry using CB (12.9\%) was selectively performed in malignancy-suspicious and equivocal cases (Table S1). The distribution of diagnoses differed according to the procedures (Table S2). LBC detected $78.8 \%$ negative, $5.5 \%$ atypical, $2.4 \%$ suspicious, and $13.3 \%$ malignant cases. The percentage of negative cases detected by $\mathrm{CB}$ (74.9\%) and the combination of CB and LBC $(72.9 \%)$ was slightly lower than that detected by LBC, whereas the opposite pattern was observed in malignant cases $(17.2 \%$ and $18.2 \%$, respectively). Among cases examined using CEA immunostaining, 74\% were malignant cases because immunocytochemistry was not implemented in the definite negative cases (Table S2). 
Table 1 Discrepancy between LBC and CB

\begin{tabular}{|c|c|c|c|c|c|c|}
\hline LBC & \multicolumn{6}{|c|}{$\mathrm{CB}$} \\
\hline Negative, n (\%) & 628 (93.2) & $15(31.9)$ & $9(37.5)$ & $22(14.2)$ & $94(82.5)$ & 768 \\
\hline Atypical, n (\%) & $20(3.0)$ & $19(40.4)$ & $1(4.2)$ & $7(4.5)$ & $7(6.1)$ & 54 \\
\hline Suspicious, n (\%) & $5(0.7)$ & $6(12.8)$ & $1(4.2)$ & $7(4.5)$ & $4(3.5)$ & 23 \\
\hline Not available, n (\%) & $18(2.7)$ & 7 (14.9) & $1(4.2)$ & $13(8.4)$ & - & 39 \\
\hline Total, $\mathrm{n}$ & 674 & 47 & 24 & 155 & 114 & 1,014 \\
\hline
\end{tabular}

LBC, liquid based cytology; CB, cell block.

Table 2 Discrepancy between CB and CEA immunostaining

\begin{tabular}{lccc}
\hline \multirow{2}{*}{ CB } & \multicolumn{2}{c}{ CEA immunostaining } & \multirow{2}{*}{ Total } \\
\cline { 2 - 3 } & Negative & Positive & \\
\hline Negative, n (\%) & $1(2.9)$ & $3(3.1)$ & $4(3.1)$ \\
Atypical, n (\%) & $16(47.1)$ & $5(5.2)$ & $21(16.0)$ \\
Suspicious, n (\%) & $6(17.6)$ & $7(7.2)$ & $13(9.9)$ \\
Malignant, n (\%) & $11(32.4)$ & $82(84.5)$ & $93(71.0)$ \\
Total, n & 34 & 97 & 131 \\
\hline
\end{tabular}

$\mathrm{CB}$, cell block; CEA, carcinoembryonic antigen.

\section{Primary sites of MPE}

A total of 206 samples (20.3\%) tapped from 182 patients (21.1\%) were classified as "diagnosis confirmed malignant". The most common organ was the lung $(72.8 \%)$, and $>50 \%$ of confirmed malignant cases were identified as lung adenocarcinoma $(58.7 \%)$. The common causes of MPE were as follows: lung small cell carcinoma $(8.7 \%)$, breast cancer $(6.8 \%)$, gastric cancer (4.4\%), lung squamous cell carcinoma (3.9\%), and colorectal cancer (3.4\%) (Table S3).

\section{Discrepancy between $L B C, C B$, and CEA immunostaining}

Except for equivocal cases (atypical), 39 cases showed a discrepancy between LBC and CB (Table 1). Among LBC cases classified as negative, CB analysis identified 22 $(3.3 \%)$ as malignant, $9(1.3 \%)$ as suspicious, and $15(2.2 \%)$ as atypical. Among the cases identified as malignant by LBC, three $(2.5 \%)$ were negative on CB. The 5 (26.3\%) and $6(31.6 \%)$ cases diagnosed as suspicious on LBC were negative and atypical on $\mathrm{CB}$, respectively (Table 1).
CEA immunostaining identified 19 (63.3\%) of 30 LBC negative cases as positive and $4(23.5 \%)$ of 17 equivocal cases as positive. Eight cases of LBC including two suspicious and six malignant did not show immunoreactivity (Table S4). In CB preparations, 3 (75\%, 3/4) negative and $5(23.8 \%, 5 / 21)$ atypical cases were CEA positive. Eleven malignant cases by CB $(11.8 \%, 11 / 93)$ were immunonegative (Table 2). CEA-positive cases were confirmed as malignancy. However, despite the definite malignant cases, CEA was negative in $6 \mathrm{LBC}(7.9 \%)$ and $11 \mathrm{CB}(11.8 \%)$ cases.

Based on the cellular morphology of $\mathrm{CB}$ preparations and the results of CEA immunostaining, 22 (73.3\%) of 30 negative cases by LBC were regrouped as malignant. Similarly, 5 atypical LBC (29.4\%) cases were re-categorized as malignant, and 12 atypical (70.6\%) cases were redefined as negative (Table S5). After CEA immunostaining, 3 negative cases $(75.0 \%)$ and 5 atypical cases $(23.8 \%)$ on CB preparations were regrouped as malignant, whereas 16 atypical (76.2\%), 4 suspicious (30.8\%), and 2 malignant cases $(2.2 \%)$ were redefined as negative (Table 3 ).

\section{Discrepancy between cytology and final diagnosis}

Of the confirmed malignant cases, 129 (68.3\%) LBC specimens were interpreted as malignant, $19(10.1 \%)$ as suspicious, $7(3.7 \%)$ as atypical, and $34(18.0 \%)$ as negative, whereas $150(78.9 \%)$ CB samples were diagnosed as malignant, $20(10.5 \%)$ as suspicious, $10(5.3 \%)$ as atypical, and $10(5.3 \%)$ as negative. Two cases classified as malignant by $\mathrm{CB}$ were negative. The combination of LBC and $\mathrm{CB}$ identified $154(87.0 \%)$ cases as malignant. The proportion of confirmed malignant cases showing CEA immunoreactivity was $89.8 \%(97 / 108)$. Addition of CEA 
Table 3 Discrepancy between CB and the combined use of CB and CEA immunostaining

\begin{tabular}{|c|c|c|c|c|c|}
\hline $\mathrm{CB}$ & \multicolumn{4}{|c|}{$\mathrm{CB}$ and $\mathrm{CEA}$} & Tota \\
\hline Negative, n (\%) & $1(4.3)$ & 0 & 0 & $3(2.8)$ & 4 \\
\hline Atypical, n (\%) & $16(69.6)$ & 0 & 0 & $5(4.7)$ & 21 \\
\hline Suspicious, n (\%) & $4(17.4)$ & $2(100)$ & 0 & $7(6.6)$ & 13 \\
\hline Total, $\mathrm{n}$ & 23 & 2 & 0 & 106 & 131 \\
\hline
\end{tabular}

$\mathrm{CB}$, cell block; CEA, carcinoembryonic antigen.

immunostaining to cytological examination (CB) increased the diagnostic rate of malignancy to $98.1 \%$ (Table 4).

\section{Diagnostic accuracy of $L B C, C B, C E A$ immunostaining, and combination tests}

The cytological diagnoses were categorized except for cases diagnosed as atypical. The "negative" cases were reclassified as benign, whereas "suspicious" and "malignant" cases were reclassified as "malignant" to evaluate the sensitivity and specificity of each preparation and the combination (Tables S6-S10). CB preparations showed a better diagnostic accuracy than LBC. The sensitivity and specificity of CB preparations were estimated as $94.3 \%$ and $98.7 \%$, respectively, compared with $81.3 \%$ and $99.4 \%$ for LBC preparations, respectively. The combination of $\mathrm{LBC}$ and $\mathrm{CB}$ increased sensitivity to $98.3 \%$. Although CEA staining itself did not show superior accuracy (sensitivity, $89.8 \%$ ), the combined use of $\mathrm{CB}$ and the tumor marker increased the detection rate of malignancy (sensitivity, 100\%; specificity, $100 \%$ ), compared with that of cytology (LBC or CB) alone (Table 5).

\section{Discussion}

The use of cytology for diagnosis has certain advantages, as it is a non-invasive, simple, and inexpensive method. LBC preparations generated by an automated and standardized CellprepPlus ${ }^{\circledast}$ process facilitate the identification of malignant cells and increase the diagnostic accuracy compared with that of conventional smears. However, discriminating malignant cells among reactive mesothelial cells and macrophages in the pleural fluid is challenging using only the morphological features detected by LBC. Cancer cells in effusion fluids are less intact and less cohesive, and exist in loose clusters or as isolated single cells that lack the organoid pattern observed in primary tumors (Figure 1A). After LBC preparation, residual fluid specimens can be used in $\mathrm{CB}$ preparations for immunocytochemical and molecular studies. LBC is less sensitive than CB $(81.3 \%$ vs. $94.3 \%)$. The cellular architectural features of $\mathrm{CB}$ preparations may appear similar to those of tissue specimens, such as a glandular structure, a squamoid structure, or nuclear molding, which facilitates the differentiation of malignant cells from reactive mesothelial cells and macrophages (Figure 1B). However, cellular aggregates of reactive mesothelial cells are commonly observed in metastatic adenocarcinoma. Therefore, ancillary tests such as immunocytochemical staining of $\mathrm{CB}$ preparations contribute to the differential diagnosis.

CEA is a widely used marker that is reliable and specific for adenocarcinoma. The sensitivity of this marker in cytology highly depends on the origin of the primary tumor; however, adenocarcinomas account for a large proportion of MPEs regardless of the primary site (Figure 1C). Thus, CEA immunostaining with high sensitivity can be helpful for cytological diagnosis. In the present study, the sensitivity and negative predictive values of $\mathrm{CB}$ and CEA immunostaining were superior to those of LBC alone for detecting MPE. The combination of cytological examination and CEA increased the detection rate of malignancy by $20 \%$ and $9 \%$, compared with that of cytology alone (LBC and $\mathrm{CB}$, respectively). CEA immunoreactivity resulted in the regrouping of 82 cases (43 LBC and $39 \mathrm{CB}$ ) into 36 benign and 42 malignant cases (Table 3 and Table S5). The 38 equivocal cases $(37.6 \%, 17 \mathrm{LBC}$ and $21 \mathrm{CB})$ were reclassified into 28 benign and 10 malignant cases. Some benign cases confirmed by CEA immunonegativity showed morphological features similar to those of cancer cells, such as large nuclei, cellular aggregates, and irregular 


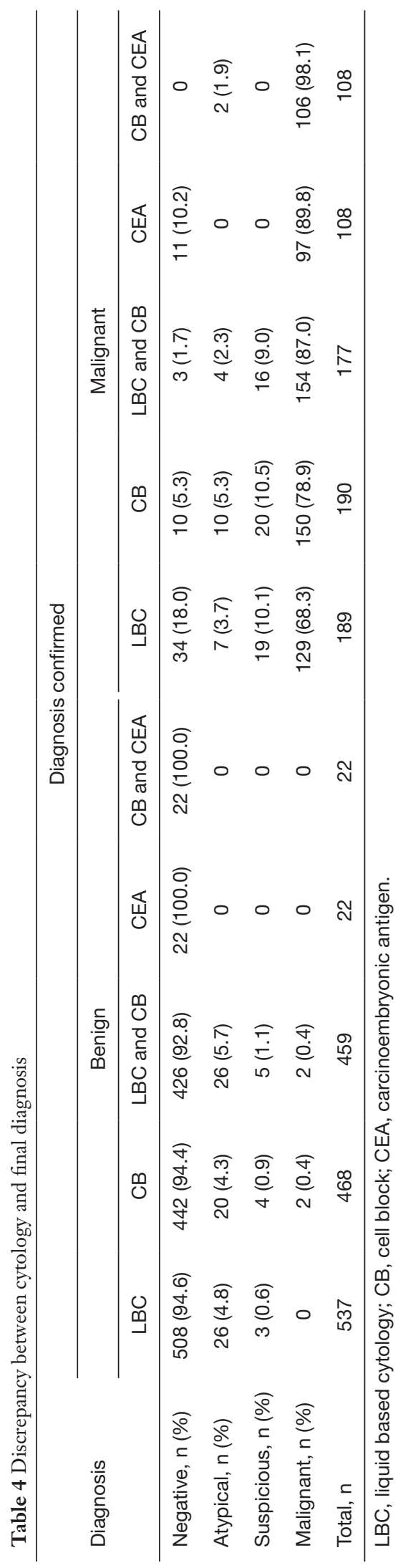

nuclear membranes. These atypical cells were reactive mesothelial cells. Nonspecific reactivity for CEA in macrophages and other inflammatory cells was reported previously $(23,24)$. However, none of the benign cases were immunopositive for CEA in the present study. CEA immunostaining is a useful approach to achieve a correct diagnosis in many questionable cases. Malignant cases with CEA negativity included seven lung adenocarcinomas $(9.9 \%, 7 / 71)$, two high grade ovarian serous carcinomas $(100 \%, 2 / 2)$, a breast invasive micropapillary carcinoma $(14.3 \%, 1 / 7)$, and a thyroid undifferentiated carcinoma. Four lung adenocarcinomas were detected by thyroid transcription factor-1 (TTF-1) immunostaining $(25,26)$, and the remaining three cases were confirmed by pleural biopsy. Two ovarian carcinoma cases showed positivity for cytokeratin 7 and PAX8 instead of CEA positivity, which is consistent with studies showing negative CEA immunoreactivity in ovarian serous carcinoma. Additional tumor markers may be necessary to verify the neoplastic origin of the primary tumor. Despite the combined analysis of CB preparations and CEA, two cases (CEA-negative) remained equivocal. In these cases, $\mathrm{CB}$ preparations revealed suspicious features, whereas definite malignant cells were identified by LBC. The primary lesions were confirmed as lung adenocarcinoma histopathologically.

We investigated the diagnostic performance of the combination of LBC and CEA immunostaining in CB preparations. The concurrent analysis of $\mathrm{LBC}, \mathrm{CB}$, and CEA immunostaining was beneficial to improve the diagnostic accuracy of cytopathology in pleural effusion based on higher sensitivity and specificity than those of morphologic features (LBC or CB) alone. These three methods are complementary. $\mathrm{CB}$ preparations preserved the architectural pattern of the primary tumor to distinguish among the equivocal cytological features of LBC slides, and CEA immunoreactivity facilitated the definite identification of positive cases $(5,12,13,21,22)$. CEA positivity is indicative of the presence of adenocarcinoma cells. Atypical cells showing CEA immunonegativity can be diagnosed as benign reactive cells. However, not all carcinomatous cells show CEA expression, and surface proteins may be degraded during CB processing; therefore, cases that show definite malignant cytological features in LBC slides and those that are clinically confirmed as malignancy should be considered malignant despite CEA negativity in CB preparations.

The present study had several limitations. First, there was a possible selection bias inherent to the retrospective nature of this single-institution study. Second, a single 
Table 5 Accuracy of each cytological method

\begin{tabular}{llllll}
\hline Accuracy & LBC & CB & LBC and CB & CEA & CB and CEA \\
\hline Sensitivity, \% (95\% Cl) & $81.3(75.0-87.6)$ & $94.3(90.8-97.8)$ & $98.3(96.4-100)$ & $89.8(83.8-95.8)$ & 100 \\
Specificity, \% (95\% Cl) & $99.4(98.7-100)$ & $98.7(97.6-99.8)$ & $98.4(97.2-99.6)$ & 100 & 100 \\
Positive predictive value, \% & 98.0 & 96.6 & 96.0 & 100 & 100 \\
Negative predictive value, \% & 93.7 & 97.8 & 99.3 & 66.7 & 100 \\
\hline
\end{tabular}

LBC, liquid based cytology; $\mathrm{CB}$, cell block; CEA, carcinoembryonic antigen; $\mathrm{Cl}$, confidence intervals.

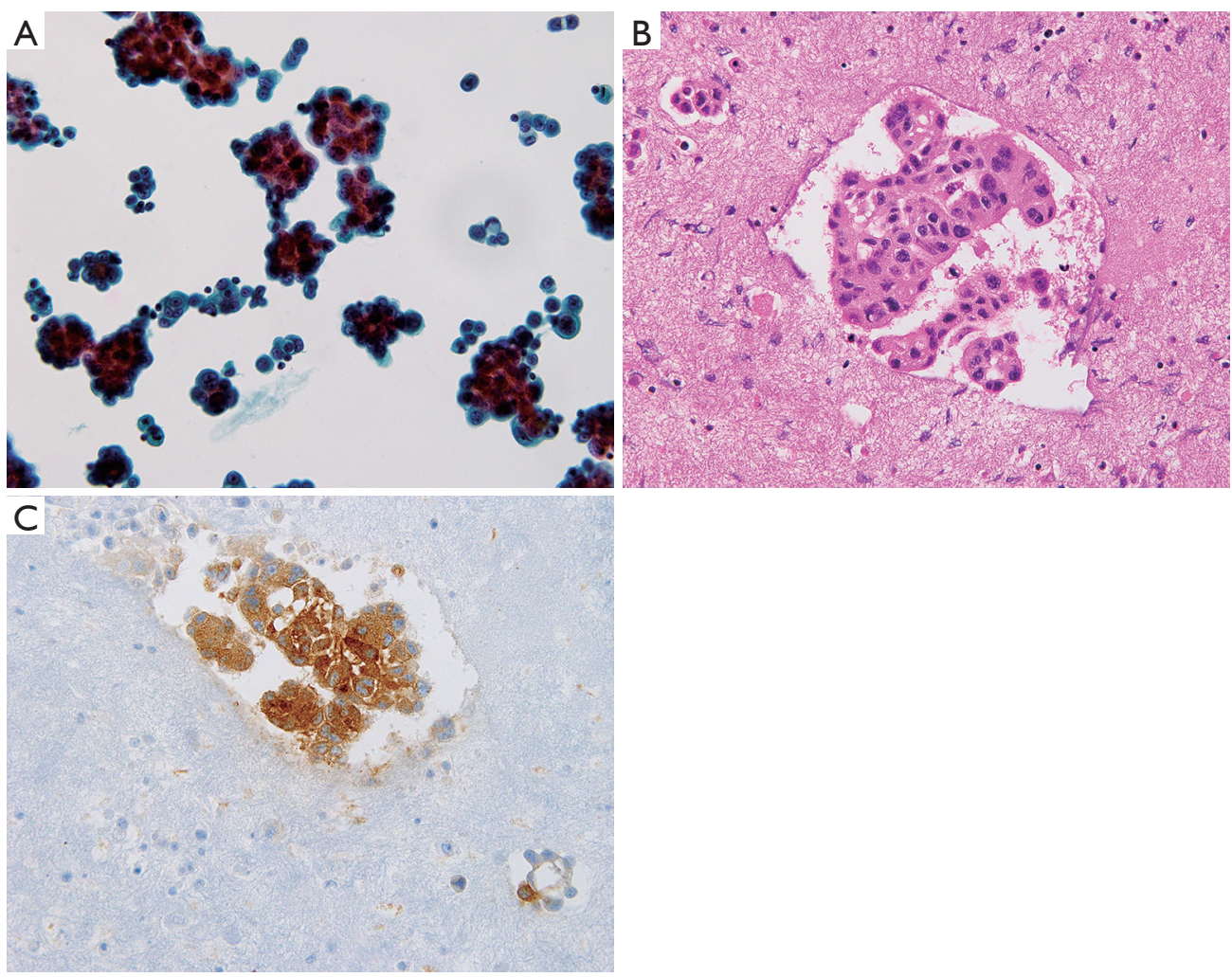

Figure 1 Cancer cells in liquid based cytology (CellprepPlus ${ }^{\circledR}$ ) are less cohesive, and exist in loose clusters or as isolated single cells (A). A cell block preparation (hematoxylin eosin \& eosin, $\times 400$ ) with a similar appearance to that of tissue specimens, such as the presence of a glandular structure appearance (B). Tumor cells are positive for CEA immunostaining using a cell block (immunocytochemistry, $\times 400)(\mathrm{C})$.

tumor marker, CEA, was analyzed. Different antibodies serve as reliable markers for the differential diagnosis of reactive mesothelial cells. Although several markers are used in immunocytochemistry, we did not include them to simplify the results and improve clarity. Finally, we did not perform immunostaining for CEA to define all subtypes of malignancies in cases showing no treatment benefit. This could also lead to a selection bias.
In summary, analysis of $\mathrm{CB}$ preparations and CEA immunostaining along with LBC showed a convincing diagnostic performance. Considering the synergistic effects, the $\mathrm{CB}$ method and CEA staining should be integrated into $\mathrm{LBC}$ in routine clinical practice to improve the diagnostic accuracy of pleural effusion, especially in cases in which malignancy is suspected or those showing equivocal cytological features. 


\section{Acknowledgements}

Funding: This work was supported by the National Research Foundation of Korea (NRF) grant funded by the Korea government (MSIT) (2017R1A2B4010169).

\section{Footnote}

Conflicts of Interest: The authors have no conflicts of interest to declare.

Ethical Statement: The present study adhered to the guidelines established by the Declaration of Helsinki and was approved by the Institutional Review Board of Chungbuk National University Hospital (2014-02-009).

\section{References}

1. Leuallen EC, Carr DT. Pleural effusion; a statistical study of 436 patients. N Engl J Med 1955;252:79-83.

2. Light RW, Macgregor MI, Luchsinger PC, et al. Pleural effusions: the diagnostic separation of transudates and exudates. Ann Intern Med 1972;77:507-13.

3. Shidham VB, Falzon M. Serous effusions. In: Gray W, Kocjan G. Editors. Diagnostic Cytopathology. Third edition. London: Churchill Livingstone, 2010;128-30.

4. Lee YM, Hwang JY, Son SM, et al. Comparison of diagnostic accuracy between CellprepPlus(R) and ThinPrep(R) liquid-based preparations in effusion cytology. Diagn Cytopathol 2014;42:384-90.

5. Dekker A, Bupp PA. Cytology of serous effusions. An investigation into the usefulness of cell blocks versus smears. Am J Clin Pathol 1978;70:855-60.

6. Sahn SA. Pleural diseases related to metastatic malignancies. Eur Respir J 1997;10:1907-13.

7. Hooper C, Lee YC, Maskell N. Investigation of a unilateral pleural effusion in adults: British Thoracic Society Pleural Disease Guideline 2010. Thorax 2010;65 Suppl 2:ii4-17.

8. Rivera MP, Mehta AC, Wahidi MM. Establishing the diagnosis of lung cancer: Diagnosis and management of lung cancer, 3rd ed: American College of Chest Physicians evidence-based clinical practice guidelines. Chest 2013;143:e142S-65S.

9. Son SM, Koo JH, Choi SY, et al. Evaluation of Urine Cytology in Urothelial Carcinoma Patients: A Comparison of CellprepPlus(R) Liquid-Based Cytology and Conventional Smear. Korean J Pathol 2012;46:68-74.
10. Zhao FH, Hu SY, Bian JJ, et al. Comparison of ThinPrep and SurePath liquid-based cytology and subsequent human papillomavirus DNA testing in China. Cancer Cytopathol 2011;119:387-94.

11. Bedrossian CW. Diagnostic problems in serous effusions. Diagn Cytopathol 1998;19:131-7.

12. Dey S, Nag D, Nandi A, et al. Utility of cell block to detect malignancy in fluid cytology: Adjunct or necessity? J Cancer Res Ther 2017;13:425-9.

13. Assawasaksakul T, Boonsarngsuk V, Incharoen P. A comparative study of conventional cytology and cell block method in the diagnosis of pleural effusion. J Thorac Dis 2017;9:3161-7.

14. Fowler LJ, Lachar WA. Application of immunohistochemistry to cytology. Arch Pathol Lab Med 2008;132:373-83.

15. Fischer AH, Schwartz MR, Moriarty AT, et al. Immunohistochemistry practices of cytopathology laboratories: a survey of participants in the College of American Pathologists Nongynecologic Cytopathology Education Program. Arch Pathol Lab Med 2014;138:1167-72.

16. Koksal D, Demirag F, Bayiz H, et al. The cell block method increases the diagnostic yield in exudative pleural effusions accompanying lung cancer. Turk Patoloji Derg 2013;29:165-70.

17. Ko EC, Jhala NC, Shultz JJ, et al. Use of a panel of markers in the differential diagnosis of adenocarcinoma and reactive mesothelial cells in fluid cytology. Am J Clin Pathol 2001;116:709-15.

18. Alatas F, Alatas O, Metintas M, et al. Diagnostic value of CEA, CA 15-3, CA 19-9, CYFRA 21-1, NSE and TSA assay in pleural effusions. Lung Cancer 2001;31:9-16.

19. Paganuzzi M, Onetto M, Marroni P, et al. Diagnostic value of CYFRA 21-1 tumor marker and CEA in pleural effusion due to mesothelioma. Chest 2001;119:1138-42.

20. Son SM, Han HS, An JY, et al. Diagnostic performance of CD66c in lung adenocarcinoma-associated malignant pleural effusion: comparison with CEA, CA 19-9, and CYFRA 21-1. Pathology 2015;47:123-9.

21. Thapar M, Mishra RK, Sharma A, et al. Critical analysis of cell block versus smear examination in effusions. J Cytol 2009;26:60-4.

22. Shivakumarswamy U, Arakeri SU, Karigowdar MH, et al. Diagnostic utility of the cell block method versus the conventional smear study in pleural fluid cytology. J Cytol 2012;29:11-5.

23. Tickman RJ, Cohen C, Varma VA, et al. Distinction between carcinoma cells and mesothelial cells in serous 
effusions. Usefulness of immunohistochemistry. Acta Cytol 1990;34:491-6.

24. Silverman JF, Nance K, Phillips B, et al. The use of immunoperoxidase panels for the cytologic diagnosis of malignancy in serous effusions. Diagn Cytopathol 1987;3:134-40.

25. Pelosi G, Fraggetta F, Pasini F, et al. Immunoreactivity for thyroid transcription factor-1 in stage I non-small cell carcinomas of the lung. Am J Surg Pathol 2001;25:363-72.

26. Elsamany SA, Al-Fayea TM, Alzahrani AS, et al. Thyroid transcription factor-1 expression in advanced non-small cell lung cancer: impact on survival outcome. Asian Pac J Cancer Prev 2015;16:2987-91.

Cite this article as: Woo CK, Son SM, Han HS, Lee KH, Choe KH, An JY, Lee KM, Lim YH, Lee HC, Lee OJ. Diagnostic benefits of the combined use of liquid-based cytology, cell block, and CEA immunocytochemistry in malignant pleural effusion. J Thorac Dis 2018;10(8):4931-4939. doi: $10.21037 /$ jtd.2018.07.139 
Supplementary

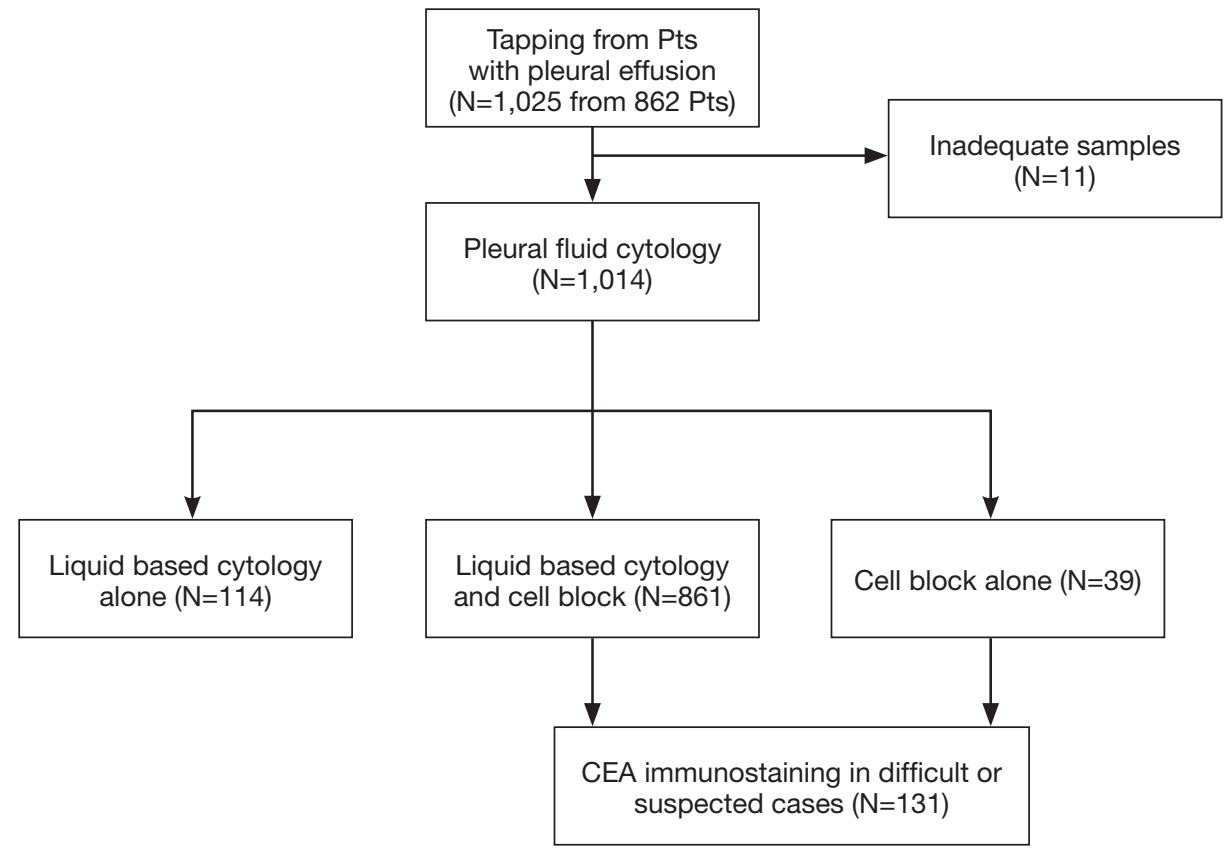

Figure S1 The CONSORT flow chart.

Table S1 Pleural fluid cytology

\begin{tabular}{lcc}
\hline Procedures & Cases & Patients \\
\hline LBC, n (\%) & $975(96.2)$ & $837(97.1)$ \\
CB, n (\%) & $900(88.8)$ & $769(89.2)$ \\
LBC and CB, n (\%) & $861(84.9)$ & $744(86.3)$ \\
CEA, n (\%) & $131(12.9)$ & $129(15.0)$ \\
Total, n & 1,014 & 862 \\
\hline
\end{tabular}

LBC, liquid based cytology; CB, cell block; CEA, carcinoembryonic antigen.

Table S2 Diagnosis according to procedures

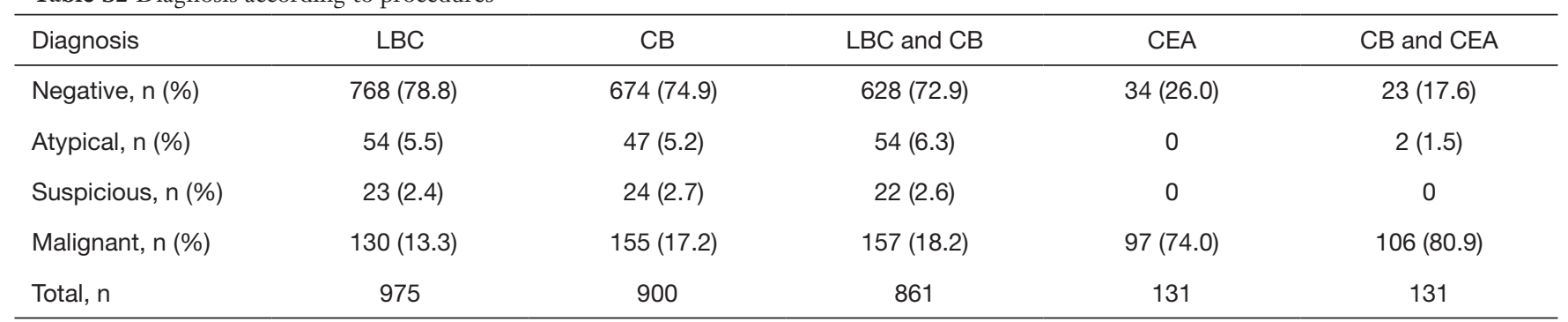

LBC, liquid based cytology; CB, cell block; CEA, carcinoembryonic antigen. 
Table S3 Primary sites of malignant pleural effusion

\begin{tabular}{lcc}
\hline Organs & Cases & Patients \\
\hline Lung, n (\%) & $150(72.8)$ & $132(72.5)$ \\
Adenocarcinoma & $121(58.7)$ & $104(57.1)$ \\
Small cell carcinoma & $18(8.7)$ & $18(9.9)$ \\
Squamous cell carcinoma & $8(3.9)$ & $7(3.8)$ \\
Others & $3(1.5)$ & $3(1.6)$ \\
Breast, n (\%) & $14(6.8)$ & $12(6.6)$ \\
Stomach, n (\%) & $9(4.4)$ & $7(3.8)$ \\
Large intestine, n (\%) & $7(3.4)$ & $7(3.8)$ \\
Ovary, n (\%) & $4(1.9)$ & $4(2.2)$ \\
Gallbladder, n (\%) & $4(1.9)$ & $4(2.2)$ \\
Lymphoma, n (\%) & $2(1.0)$ & $2(1.1)$ \\
Others, n (\%) & $11(5.3)$ & $11(6.0)$ \\
Unknown primary, n (\%) & $5(2.4)$ & $3(1.6)$ \\
Total, n & 206 & 182 \\
\hline
\end{tabular}

Table S4 Discrepancy between LBC and CEA

\begin{tabular}{lccc}
\hline \multirow{2}{*}{ LBC } & \multicolumn{2}{c}{ CEA } & \multirow{2}{*}{ Total } \\
\cline { 2 - 3 } & Negative & Positive & \\
\hline Negative, n (\%) & $11(34.4)$ & $19(20.4)$ & $30(24.0)$ \\
Atypical, n (\%) & $13(40.6)$ & $4(4.3)$ & $17(13.6)$ \\
Suspicious, n (\%) & $2(6.3)$ & 0 & $2(1.6)$ \\
Malignant, n (\%) & $6(18.8)$ & $70(75.3)$ & $76(60.8)$ \\
Total, n & 32 & 93 & 125 \\
\hline
\end{tabular}

LBC, liquid based cytology; CEA, carcinoembryonic antigen.

Table S5 Discrepancy between LBC and the combined use of CB and CEA

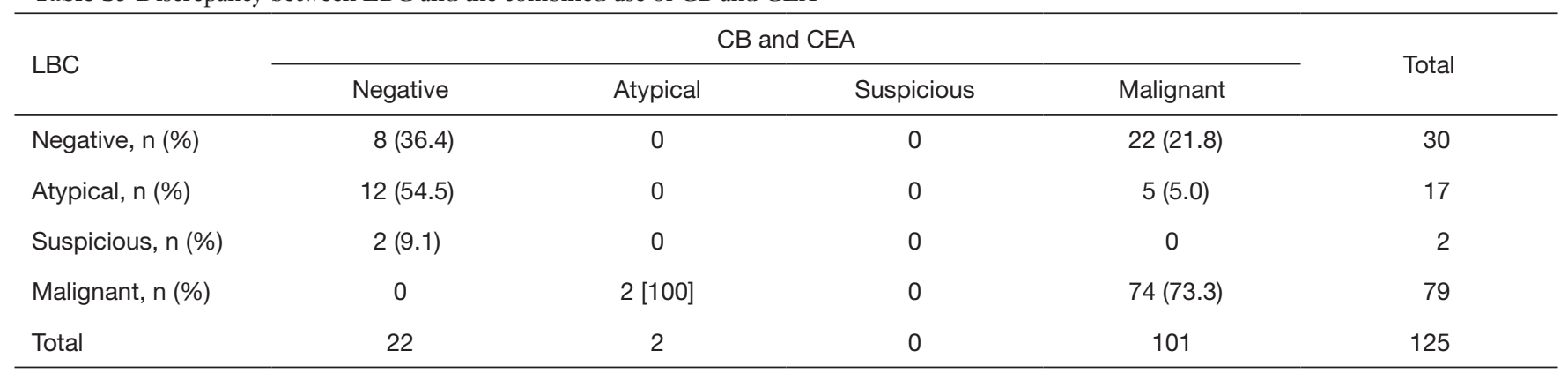

LBC, liquid based cytology; CB, cell block; CEA, carcinoembryonic antigen.

Table S6 The results of LBC according to final diagnosis

\begin{tabular}{lccc}
\hline \multirow{2}{*}{ LBC } & \multicolumn{2}{c}{ Final diagnosis } & \multirow{2}{*}{ Total } \\
\cline { 2 - 3 } & \multicolumn{1}{c}{ Benign } & Malignant & \\
\hline Benign, $\mathrm{n}(\%)$ & $508(99.4)$ & $34(18.7)$ & 542 \\
Malignant, $\mathrm{n}(\%)$ & $3(0.6)$ & $148(81.3)$ & 151 \\
Total, $\mathrm{n}$ & 511 & 182 & 693 \\
\hline
\end{tabular}

LBC, liquid based cytology.
Table S7 The results of CB according to final diagnosis

\begin{tabular}{lccc}
\hline \multirow{2}{*}{ CB } & \multicolumn{2}{c}{ Final diagnosis } & \multirow{2}{*}{ Total } \\
\cline { 2 - 3 } & Benign & Malignant & \\
\hline Benign, $\mathrm{n}(\%)$ & $442(98.7)$ & $10(5.7)$ & 452 \\
Malignant, $\mathrm{n}(\%)$ & $6(1.3)$ & $170(94.3)$ & 176 \\
Total, $\mathrm{n}$ & 448 & 180 & 628 \\
\hline
\end{tabular}

$\mathrm{CB}$, cell block. 
Table S8 The results of the combination of LBC and CB according to final diagnosis

\begin{tabular}{lccc}
\hline \multirow{2}{*}{ LBC and CB } & \multicolumn{2}{c}{ Final diagnosis } & \multirow{2}{*}{ Total } \\
\cline { 2 - 3 } & Benign & Malignant & \\
\hline Benign, $\mathrm{n}(\%)$ & $426(98.4)$ & $3(1.7)$ & 429 \\
Malignant, $\mathrm{n}(\%)$ & $7(1.6)$ & $170(98.3)$ & 177 \\
Total, $\mathrm{n}$ & 433 & 173 & 606 \\
\hline
\end{tabular}

LBC, liquid based cytology; CB, cell block.

Table S9 The results of CEA immunostaining according to final diagnosis

\begin{tabular}{lccc}
\hline \multirow{2}{*}{ CEA immunostaining } & \multicolumn{2}{c}{ Final diagnosis } & \multirow{2}{*}{ Total } \\
\cline { 2 - 3 } & Benign & Malignant & \\
\hline Negative, $\mathrm{n}(\%)$ & $22[100]$ & $11(10.2)$ & 33 \\
Positive, $\mathrm{n}(\%)$ & 0 & $97(89.8)$ & 97 \\
Total, $\mathrm{n}$ & 22 & 108 & 130 \\
\hline
\end{tabular}

CEA, carcinoembryonic antigen.

Table S10 The results of the combined use of CB and CEA immunostaining according to final diagnosis

\begin{tabular}{lccc}
\hline \multirow{2}{*}{ CB and CEA immunostaining } & \multicolumn{2}{c}{ Final diagnosis } & \multirow{2}{*}{ Total } \\
\cline { 2 - 3 } & Benign & Malignant & \\
\hline Benign, $\mathrm{n}(\%)$ & $22[100]$ & 0 & 22 \\
Malignant, $\mathrm{n}(\%)$ & 0 & $106[100]$ & 106 \\
Total, $\mathrm{n}$ & 22 & 106 & 128 \\
\hline
\end{tabular}

$\mathrm{CB}$, cell block; CEA, carcinoembryonic antigen. 